Itinéraires Itinéraires

Littérature, textes, cultures

2009-2 | 2009

Caraïbe et océan Indien

\title{
Valérie Magdelaine-Andrianjafitrimo et Jean- Claude Carpanin Marimoutou, Univers créoles 6. Le Champ littéraire réunionnais en questions
}

\section{Stéphane Hoarau}

\section{(2) OpenEdition}

Journals

Édition électronique

URL : http://journals.openedition.org/itineraires/221

DOI : 10.4000/itineraires.221

ISSN : 2427-920X

Éditeur

Pléiade

\section{Édition imprimée}

Date de publication : 1 juillet 2009

Pagination : 184-186

ISBN : 978-2-296-09639-4

ISSN : $2100-1340$

Référence électronique

Stéphane Hoarau, « Valérie Magdelaine-Andrianjafitrimo et Jean-Claude Carpanin Marimoutou, Univers créoles 6. Le Champ littéraire réunionnais en questions », Itinéraires [En ligne], 2009-2 | 2009, mis en ligne le 22 septembre 2014, consulté le 22 septembre 2020. URL : http://journals.openedition.org/ itineraires/221; DOI : https://doi.org/10.4000/itineraires.221

Ce document a été généré automatiquement le 22 septembre 2020.

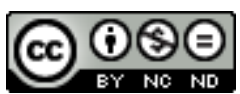

Itinéraires est mis à disposition selon les termes de la licence Creative Commons Attribution - Pas d'Utilisation Commerciale - Pas de Modification 4.0 International. 


\title{
Valérie Magdelaine- Andrianjafitrimo et Jean-Claude Carpanin Marimoutou, Univers créoles 6. Le Champ littéraire réunionnais en questions
}

\author{
Stéphane Hoarau
}

\section{RÉFÉRENCE}

Valérie Magdelaine-Andrianjafitrimo et Jean-Claude Carpanin Marimoutou, Univers créoles 6. Le Champ littéraire réunionnais en questions, Paris, Economica-Anthropos, 2006, 176 pages, ISBN : 978-2-717-85170-0.

Ce numéro d'Univers créoles, rédigé par Valérie Magdelaine-Andrianjafitrimo et JeanClaude Carpanin Marimoutou, tous deux enseignants-chercheurs à l'université de l'île de La Réunion, propose de faire le point des connaissances se rapportant au champ littéraire réunionnais. L'ouvrage se découpe en quatre chapitres, le quatrième comprenant une importante bibliographie regroupant l'ensemble des œuvres réunionnaises parues entre 1994 et 2004. Celle-ci montre que sur une période de dix ans, concernant lîle de La Réunion, un peu plus de trois cents publications ont vu le jour. Les auteurs réunionnais seraient donc dynamiques. Une lecture attentive de cette bibliographie permet toutefois de constater qu'ils n'échappent pas à une sorte de dissémination, tant éditoriale (les livres sont édités le plus souvent dans l'île même et plus rarement en France, que ce soit à compte d'éditeur ou d'auteur) que générique (romans, recueils de contes, de nouvelles, de poésies, littérature jeunesse, etc.). Au vu de cette variété de références qui concerne dix années de production, on comprend 
qu'il ait paru nécessaire de se pencher sur la littérature réunionnaise pour en préciser les axes et les enjeux.

Pour ce faire, les auteurs se sont partagé la tâche comme suit: dans les deux premiers articles, Valérie Magdelaine-Andrianjafitrimo revient sur deux formes littéraires de l'île, le roman-feuilleton, puis le récit de vie. Dans un troisième article, Jean-Claude Carpanin Marimoutou se charge de questionner les textes d'une production culturelle (musicale) qui, comme il le précise, n'est pourtant pas considérée comme « littéraire », à savoir le maloya. Nous voyons ainsi, dès le sommaire, se profiler l'ambition des auteurs : non pas nécessairement étudier des formes reconnues de la littérature (roman et poésie par exemple, pourtant très présents, voire prisés par les auteurs de l'île), mais questionner des formes alternatives, souvent pensées comme étant des «sous-» ou « contre- » littératures. Il ne s'agit pas d'étudier le champ littéraire réunionnais dans sa globalité, ce qui relèverait d'une entreprise généralisante, mais de proposer des études précises portant sur divers espaces de ce champ littéraire spécifiques au $\mathrm{Xx}^{\mathrm{e}}$ siècle.

Ce qui est mis en jeu dans cet ouvrage, c'est donc moins la connaissance globale d'une littérature réunionnaise contemporaine que l'analyse d'ouvrages qui, pour le moment, n'ont vraisemblablement que très peu été étudiés : ces littératures-piliers qui ont conduit un public à la lecture et qui ont contribué à poser des jalons pour l'avenir.

Il y a d'abord, comme le rappelle déjà le titre du premier article de l'ouvrage, « Romanfeuilleton et roman en feuilleton, contribution à l'émergence de la littérature réunionnaise " (1-54), ces formes de littérature qui remettent en cause les canons littéraires (entendons : d'une "métropole»), et qui ainsi permettent de donner le jour à de nouvelles modalités d'expression en accord avec la réalité locale. Car le feuilleton, s'adaptant à un mode de circulation et à des vecteurs locaux (la presse quotidienne, etc.), réinvente ses propres codes à partir - et pour - des perspectives locales: "puisqu'il n'y a pas de consensus entre ce qu'apprend l'école française, et ce que montre le réel réunionnais » (25), le roman-feuilleton réunionnais propose un espace de négociation pour cette culture. Ce qui n'existe pas ailleurs (dans les romans français, par exemple), trouve ici un espace où se dire, où vivre au quotidien.

5 Nous retrouvons cette problématique déclinée d'une autre manière (et par là même prolongée) dans le second article, "Récits de vie, "romans mémoriels", "récits mémoriels" à La Réunion : récits et mythes du "tan lontan" " " (55-100). Là encore, il est question de l'émergence dans l'île d'une forme littéraire inédite, puisqu'il s'agit de questionner un corpus qui, à défaut de préserver une "authenticité » pourtant recherchée, tend à créer un répertoire des pratiques et des modes de vie locaux. À plusieurs reprises, Valérie Magdelaine-Andrianjafitrimo emploie le terme d'« ethnotexte » pour souligner le fait que l'abondance des descriptions présentes dans ces récits relatant les temps anciens (d'avant la départementalisation de 1946), principalement sur des modes paradoxalement nostalgiques, a pour effet d'entériner une double perte: d'abord celle des pratiques et des valeurs d'antan, puisque le discours sur le « tan lontan » postule de facto que tout ce qui a trait à ce «tan » n'existe plus ; et ensuite celle d'un présent : « le présent n'est vécu que sur le mode de la perte, à commencer par la perte de son humanité que La Réunion moderne inflige au monde créole traditionnel » (89).

6 Mais, en définitive, ce qui est véhiculé dans ces deux premiers articles, et que vient confirmer le troisième, «Le texte du maloya » par Jean-Claude Carpanin Marimoutou (101-156), c'est l'idée que ces formes de la littérature réunionnaise du $\mathrm{xx}^{\mathrm{e}}$ siècle 
contribuent « à édifier une représentation du réel réunionnais et à l'offrir à un lecteur avec lequel il veut tisser un lien » (26). Autrement dit, elles s'énoncent «pour donner lieu à d'autres récits que ceux des maîtres ", et ce, afin de s'emparer, par le discours, du lieu en question; afin de s'approprier le lieu, et d'en devenir les « maîtres » (152). C'est ce que relève Jean-Claude Carpanin Marimoutou dans les textes des maloyas traditionnels, comme dans ceux d'auteurs plus récents qui, tous, «intègrent à leur trame narrative et énonciative des fragments, des traces, des rappels des légendes et des rites venus [d'ailleurs]» (147), pour faire du chant en perpétuelle construction un «espace des héritages et des mémoires partagées » (141). Ainsi, ils parviennent à restituer le lien avec ces autres humanités séminales de l'île (autres qu'européenne), celles de Madagascar et du Tamil Nadu (Inde du Sud) par exemple.

7 Cet ouvrage questionne les modalités d'appropriation discursive du lieu et de sa mémoire par le biais de trois formes littéraires transversales (roman-feuilleton, littérature intimiste, et chants). Dans la mesure où ces modalités d'expression culturelle ont participé à élaborer un discours réunionnais apte à prendre en compte les particularités de l'île, il semblait nécessaire de revenir sur ces trois points pour en préciser les enjeux ainsi que les perspectives. Depuis la départementalisation de 1946 et donc depuis que les habitants de La Réunion sont officiellement devenus des citoyens français - ces textes, soulignent Valérie Magdelaine-Andrianjafitrimo et JeanClaude Carpanin Marimoutou, ont "vis[é] avant tout à créer un monde et un discours réunionnais » (31). C'est-à-dire, in fine, un monde et un discours qui puissent prendre en charge la totalité des liens qui ont façonné - et façonnent encore - l'identité insulaire d'aujourd'hui, non seulement dans son rapport à une histoire nationale, mais également dans un rapport conjoint à d'autres histoires - d'Afrique, d'Asie, des îles india-océanes alentour, etc.

\section{NOTES}

1. Le « tan lontan » (créole réunionnais) signifie le temps d'avant, les temps anciens...

\section{AUTEURS}

\section{STÉPHANE HOARAU}

Maison des Civilisations et de l'Unité Réunionnaise 